\title{
Modeling an emergency medical services system using computer simulation
}

\author{
Syi Su ${ }^{\mathrm{a}, *}$, Chung-Liang Shih ${ }^{\mathrm{b}}$
}

\author{
a Institute of Health Care Organization Administration, School of Public Health, National Taiwan \\ University, No. 1, Sec. 1, Jen Ai Road, Rm. 1512, Taipei 100, Taiwan, ROC \\ ${ }^{\mathrm{b}}$ Department of Emergency Medicine, National Taiwan University Hospital, No. 1, Sec. 1, \\ Jen Ai Rd, Rm. 1512, Taipei 100, Taiwan, ROC
}

Received 29 January 2003; received in revised form 19 August 2003; accepted 21 August 2003

\section{KEYWORDS}

Reallocation;

Computer simulation;

Emergency medical

service;

Prehospital care;

Utilization

\section{Introduction}

Prehospital care is an important issue in the emergency services system. There is much documen-

\footnotetext{
*Corresponding author. Tel.: +886-11-2-2341-2520; fax: +886-11-2-2341-2520.

E-mail address: susyi@ha.mc.ntu.edu.tw (S. Su).
}

\begin{abstract}
Summary Study objectives: In the emergency medical services (EMS) system, appropriate prehospital care can substantially decrease casualty mortality and morbidity. This study designed a simulation model, evaluated the existing EMS system, and suggested improvements. Methods: The study focused on 23 networked EMS hospitals affiliated with 36 emergency response units (subgroups) to perform two-tier rescues (advanced life support [ALS] in addition to basic life support [BLS] services) in Taipei, Taiwan. Using the existing EMS model as a base, this research constructed a computer simulation model and explored several model alternatives to achieve the study's objectives. The virtual models varied with staffing level, number of assigned emergency network hospitals, and various two-tier rescue probabilities. Results: Increasing the staffing to two teams for Hospital 22 lessened the call waiting probability (delay between rescue call and ambulance dispatch) by $50 \%$, even if the dispatch rate of the two-tier rescue increased from the empirical $2 \%$ to a simulated 10 and $20 \%$. Changing the two-tier rescue pattern so each EMS subgroup cooperated with two specific, preassigned network hospitals lowered the probability of patients having to wait for rescue dispatch to under $1 \%$. Conclusion: The following alternatives provided the greatest combination of effectiveness, quality patient care, and cost-efficiency: (1) because of its unique location, increase Hospital 22's staffing level to two ALS teams. (2) Establish a specific rescue protocol for the two-tier system that preassigns two network hospitals to each of the 36 EMS subgroups along with a prearranged calling sequence. If implemented, this will improve EMS performance, streamline the system, reduce randomness, and enhance efficiency.

๑) 2003 Elsevier Ireland Ltd. All rights reserved.
\end{abstract}

tation in the literature that appropriate prehospital care can significantly decrease the mortality and morbidity of casualties. The literature reveals that the survival rate for those non-traumatic cardiac arrest patients can be raised substantially through appropriate prehospital care $[1-8]$. The possible impact factors range from the prehospital care intervention time, the execution of cardiac pulmonary resuscitation (CPR) by bystanders, 
population density, the ambulance transportation distance, the execution of automatic external defibrillators and the emergency services models [4,9-11]. Cummins pointed out that for cardiac arrest clients every minute delayed on rescue intervention decreases the survival rate by $7-10 \%$ [12]. Cummins et al. also observed that if CPR was executed within $5 \mathrm{~min}$ of cardiac arrest and defibrillation occurred within $10 \mathrm{~min}$ of cardiac arrest, the percentage of survival until hospital discharge was $30 \%$; otherwise, it was less than $7 \%$ [13]. As to the impact of the emergency services model on the survival rate of those cardiac arrest patients, Eisenberg et al. mentioned that if basic life support (BLS) technicians were assigned for the prehospital rescue, the success rate was about 2-9\%. If advanced life support (ALS) technicians were assigned instead, the survival rate may reach 7-18\%. Moreover, if basic life support technicians can cooperate with advanced life support technicians to serve as a two-tier rescue model, then the survival rate can be expected to reach 4-26\% [9]. Nichol et al. evaluated the cost-effectiveness of various emergency services models from society's viewpoint and explored the method where BLS technicians provide the rescue service at the first tier and ALS technicians provide the rescue at the second tier. Such a two-tier rescue model was far more cost-effective than the one-tier one [14]. Although research showed that the two-tier rescue model has a higher survival rate than the one-tier rescue model for prehospital cardiac arrest patients, the question "Which model is best?" does not have a definite answer. Different countries and regions, based on their resource availability and specificity, developed their own rescue model. Developing countries, particularly, may equip a complex ambulance transportation system to provide rescue services. However, no matter which kind of emergency services system it is, the expenditure is not low. Hence, it is the policy maker's goal to develop and provide a cost-effective emergency rescue model that meets the population's demand despite limited resources.

The United States developed its emergency services system in the mid-1960s. The 1973 Emergency Services Act defined emergency services as "[a] system that provides for the arrangement of personnel, facilities, and equipment for the effective and coordinated delivery of health care services under emergency conditions and that is administered by a public or non-profit entity with authority and the resources to provide effective administration of the system" [15]. In 1981, the emergency casualties rescue codes and ambulance management rule was enacted in Taiwan. This was the beginning of Taiwan's emergency services system. In 1995, the Emergency Services Law was passed and hence acted as a prehospital rescue basis. Until now, most areas in Taiwan operated the EMS model mainly with a one-tier system that was staffed with BLS teams (Level I emergency medical technicians [EMTs]) providing rescue for traffic accidents, traumatic injuries, emergency diseases, intoxication accidents, etc.; however, the $2-6 \%$ survival rate of prehospital cardiac arrest patients was far lower than the foreign countries' rate of $2-44 \%$ [16]. Thus, the Taipei municipal government selected a number of specially qualified hospitals to form an emergency care hospital network with responsibility for the entire urban area. The secondary tier rescue model provided by these network hospitals can cooperate with the original first-tier BLS rescue model provided by the fire department. Thus, jointly, they can gradually establish the two-tier rescue model. The cost-effectiveness of this two-tier system should be carefully evaluated to provide a reference for future EMS model planning.

It was the goal of this study to devise an EMS model to provide the desired high performance at the lowest possible resources.

\section{Material and methods}

\subsection{Overview}

This research utilized a computer simulation method and took the EMS model of the subject system, the Taipei Emergency Medical Services System (TEMSS), as a base. We constructed a simulation model and manipulated the model's parameters and outcome analysis to identify optimum conditions. Analysis and evaluation revealed the efficiency of the two-tier rescue model. The model proposed several alternatives that may enhance the performance.

\subsection{The Taipei Emergency Medical Services System}

Taipei is located in northern Taiwan and is a highly industrialized city with an area of $272 \mathrm{~km}^{2}$, a population of 2.6 million, and 12 administrative districts. From those hospitals with special capabilities to handle emergency services, 23 were selected to form a network responsible for all 12 administrative districts. One or two network hospitals were assigned to each district to provide treatment of prehospital patients. The network hospitals cooperate with the Municipal Fire Department's 36 
affiliated emergency service subgroups within the districts to make up the Taipei Emergency Medical Services System (TEMSS). The TEMSS had used the two-tier rescue model since June 1999. Emergency medical technicians from the fire department act as the first tier to provide basic life support. Hospital medical care personnel act as the second tier and integrate with the first tier to provide advanced life support services.

\subsection{Two-tier rescue system procedure}

\subsubsection{Basic procedure}

Whenever the 119 Rescue Center gets an incident rescue call, the rescue center dispatcher obtains details of the incident (which could be, for example, prehospital cardiac arrest, chest pain, falls, trauma, poisoning, etc.) and make a spur-of-the-moment decision whether advanced life support techniques should intervene in this specific prehospital rescue event. If so, the nearest EMS subgroup's response unit (staffed with two BLS EMTs) is dispatched to the rescue task. Simultaneously, the rescue center informs the designated network hospital to send medical care personnel to participate in the event-site rescue. The first-tier response unit offers primary assessment and basic management but still has to wait for the second-tier medical team's arrival. After an advanced assessment and treatment, the casualties are then transported to the assigned network hospital. The first-tier team accompanies the second-tier rescue team to the emergency department. The teams report the process and transfer the casualties to the hospital. The first-tier team may then return to its base to be available for the next response.

If the dispatcher judges the incident a general case (not requiring advanced life support techniques), he or she dispatches the EMTs of the BLS response unit to the event site. Through their on-site assessment and primary treatment, they independently send the casualties to the assigned network hospital for advanced medical care treatment.

\subsubsection{Current system details}

Under the current system, generally each EMS subgroup cooperates with three hospitals; however, a single hospital may have up to 13 subgroups within its jurisdiction. Thus, hospitals located in the borderline of two areas may end up with too many assignments. This makes the dispatcher's job of selecting hospitals complex. The system does not run smoothly and rapidly. The irregular dispatching pattern of responsible hospitals makes the system confusing, depending highly on the dispatcher's experience. No established calling sequence or pro- tocol exists. Each hospital has one ALS rescue team available. If this team is busy and a new rescue call comes in, patients may have to wait, or the dispatcher may call another hospital. It rarely happens that both of the two assigned hospitals are busy, but if they are, the dispatcher may call a third hospital.

This aim of the study was to streamline and balance the system; to determine the optimal solution of preassigning specific, responsible hospitals to each EMS to speed up and enhance the system; and to fulfill rescue errands with the lowest cost and yet decrease in response time while reducing call waiting probability (delay in rescue dispatch).

\subsection{Model building}

This research utilized fourth generation, objectoriented simulation software, eM-Plant [17], to build the model and took the Taipei Emergency Services System as the subject. The steps and methods were as follows:

1. Clarification of the operational flowchart of the subject system: Interviews were conducted with three specialists familiar with the complete rescue model of both tiers of the subject system (see Fig. 1).

2. Data collection and analysis: The prehospital rescue records including $B L S$ and ALS information dating from 1-31 December 2000, were collected. The content covered information such as name of the response unit, time dispatch notified, time unit notified, time unit responds, time of arrival at scene, time at which the unit left the scene, time of arrival at destination hospital, time at which the unit left the hospital, and time back in service [18]. The time interval between every two event segments for a rescue event was counted to determine the following: preparation time (PRT, interval between the time the unit is notified and the time it responds), response time (RT, interval between the time the unit responds and the time it arrives at the scene), processing time (PCT, interval between the time the unit arrives at the scene and the time it leaves the scene), transport time (TT, interval between the time the unit leaves the scene and arrives at hospital), caring time (CT, interval between the time the unit arrives at the hospital and the time it leaves the hospital), and departure time (DT, interval between the time the unit leaves the hospital and the time the unit is back in service for the next rescue call) (see Fig. 1). The input variables and definitions were summarized in Table 1. BestFit software [19] was utilized to 


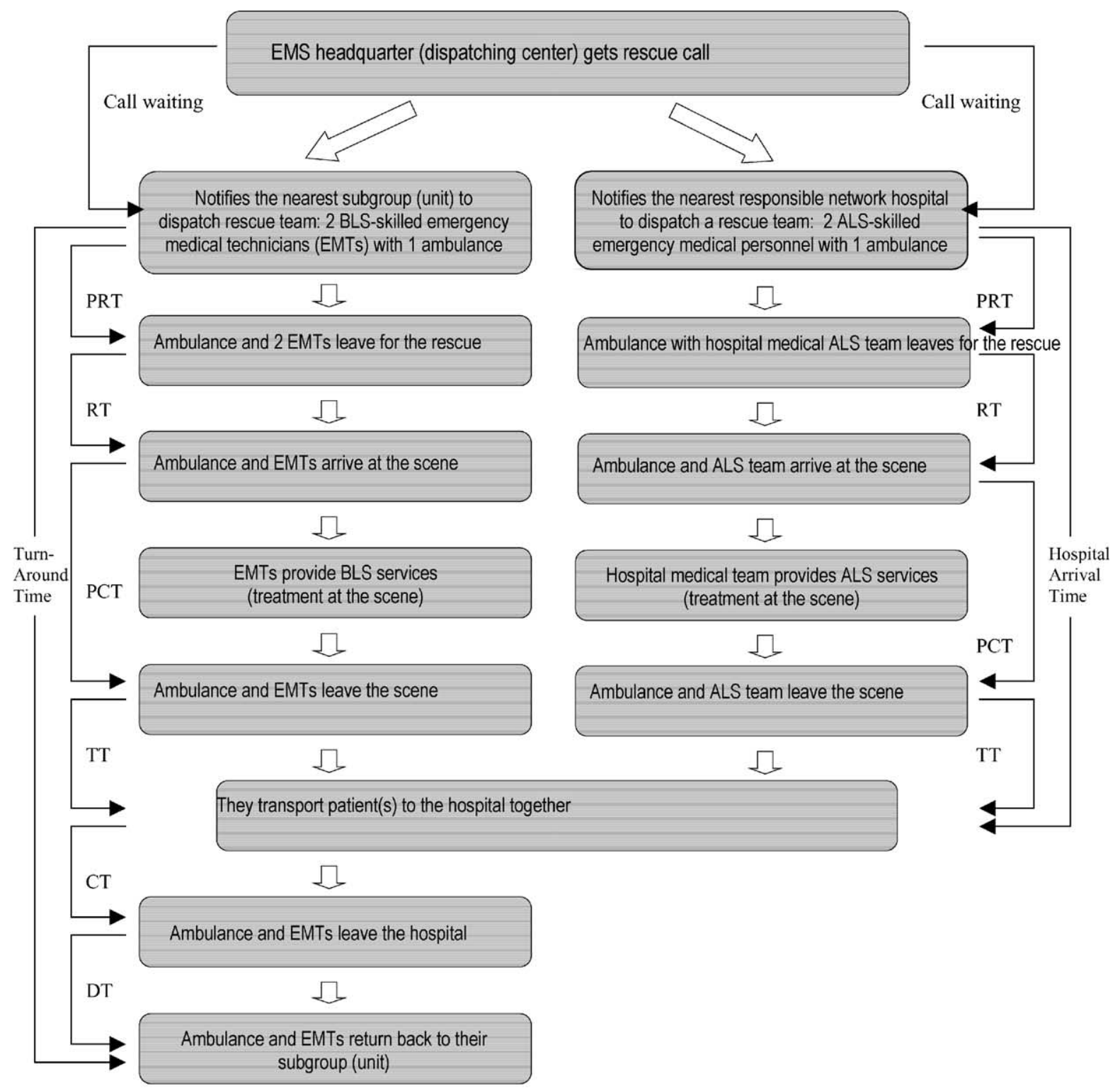

Remarks:

Two-tier rescue is required for pre-hospital cardiac arrest, trauma, dyspnea, unconsciousness, etc. When getting a rescue call, the dispatch center has to decide whether to activate a two-tier rescue pattern or just a one-tier rescue pattern.

Fig. 1 Two-tier rescue pattern-a complete turn-around. EMS: emergency services; BLS: basic life support; ALS: advanced life support; EMT: emergency medical technician. Call waiting time: interval between patient's emergency call, the dispatcher assigning a specific EMS subgroup (with corresponding network hospital) to the rescue, plus any potential waiting time if the ambulance rescue team is currently occupied with another job. Hospital arrival time: period between dispatching call and patient's hospital arrival (includes the on-site rescue time). Turn-around time: interval between the dispatching call and the BLS team's return back to its subgroup. Preparation time: PRT, interval between the time the rescue unit is notified and the time it responds. RT: response time, interval between the time the unit responds and the time it arrives at the scene. РCT: processing time, interval between the time the unit arrives at the scene and the time it leaves the scene. TT: transport time, interval between the time the unit leaves the scene and arrives at hospital. СT: caring time, interval between the time the unit arrives at the hospital and the time it leaves the hospital. DT; departure time, interval between the time the unit leaves the hospital and the time the unit is back in service for the next rescue call. 
Table 1 Input variables and definitions

\begin{tabular}{|c|c|}
\hline Input variables & Definitions \\
\hline $\begin{array}{l}\text { Inter-arrival time } \\
\text { distribution }\end{array}$ & $\begin{array}{l}\text { Probability distribution of inter-arrival time which two successive rescue calls were placed } \\
\text { to the dispatch center }\end{array}$ \\
\hline Arrival distribution & Probability distribution of time which dispatch center was notified by rescue call \\
\hline Preparation time & Interval between the time the unit is notified and the time it responds \\
\hline Response time & Interval between the time the unit responds and the time it arrives at the scene \\
\hline Processing time & Interval between the time the unit arrives at the scene and the time it leaves the scene \\
\hline Transport time & Interval between the time the unit leaves the scene and arrives at hospital \\
\hline Caring time & Interval between the time the unit arrives at the hospital and the time it leaves the hospital \\
\hline Departure time & $\begin{array}{l}\text { Interval between the time the unit leaves the hospital and the time the unit is back in } \\
\text { service for the next rescue call }\end{array}$ \\
\hline
\end{tabular}

select the best-fitting distribution of the above times. Then, the corresponding distributions were fed into the simulation model-building program. This included the following: dispatch frequency, type of rescue (one-tier or two-tier), utilization distribution of the two rescue types, and percentage of each assigned network hospital as a rescue destination.

3. Establishment of the basic model: Based on the previously confirmed prehospital rescue flowchart, the system simulation model was developed by eM-Plant as depicted in Figs. 2 and 3. This simulation software is an entirely object-oriented program using object-oriented graphic and integrated modeling for simulating and visualizing systems and business processes. Its users have the following advantages: (1) can built hierarchical models either from bottom-up or top-down; (2) can reuse of submodels or part of models stored in engineering data base; (3) can use several modeling techniques for different levels to verify the results of each level and increase productivity dramatically; (4) can centralize and define changes of all relevant system's parameters in one table; (5) can real time integrate with other software. Its input data were distributions of event inter-arrival time, preparation time, response time, processing time, transport time, caring time, and leave time (departure from the subgroup for a rescue) or idle errand (IE) time (time interval between departure from the subgroup and return to the subgroup without fulfilling a rescue). The two-tier rescue probability and distribution types of the corresponding time periods copied the probability distribution types of the historical data. The outcome variables included the utilization rates of each ambulance/rescue team, each subgroup, and the clients' waiting time (time between event report call and rescue team's arriving). Different random num- ber seeds were utilized to replicate the event 100 times in order to derive the outcome variables.

4. Validation and verification of the simulation model: (1) The established simulation model was presented to experts familiar with prehospital rescue operations to confirm the correctness and logic of the system flow. (2) Experts of simulation software and programming were requested to verify the developed simulation model. (3) The simulation outputs were compared with the empirical data to validate the model.

5. Analysis of output data: Potential alternatives were proposed to improve system performance. Each alternative was simulated, and the corresponding performances were observed and compared. Significant performance variables included the following: (1) call waiting (a call for ambulance dispatch that awaits execution). A rescue delay occurs when a hospital receives a dispatch order and has no ALS ambulance team available to go to the rescue because it is occupied with another job. Call waiting time (interval between patient's emergency call, the dispatcher assigning a specific EMS subgroup [with corresponding network hospital] to the rescue, plus any potential waiting time if the ambulance rescue team is currently occupied with another job). (2) ALS and BLS event-site arrival times (interval between the dispatching call and arrivals of the first and second tier teams on-site). (3) Hospital arrival time. (Period between the dispatching call and the patient's hospital arrival. It includes the on-site rescue time.) (4) Ambulance utilization rate (percentage of time the ambulance is being used instead of being idle). (5) Turn-around time (interval between the dispatching call and the BLS team's return back to its subgroup). The following alternatives were analyzed in sequence: 


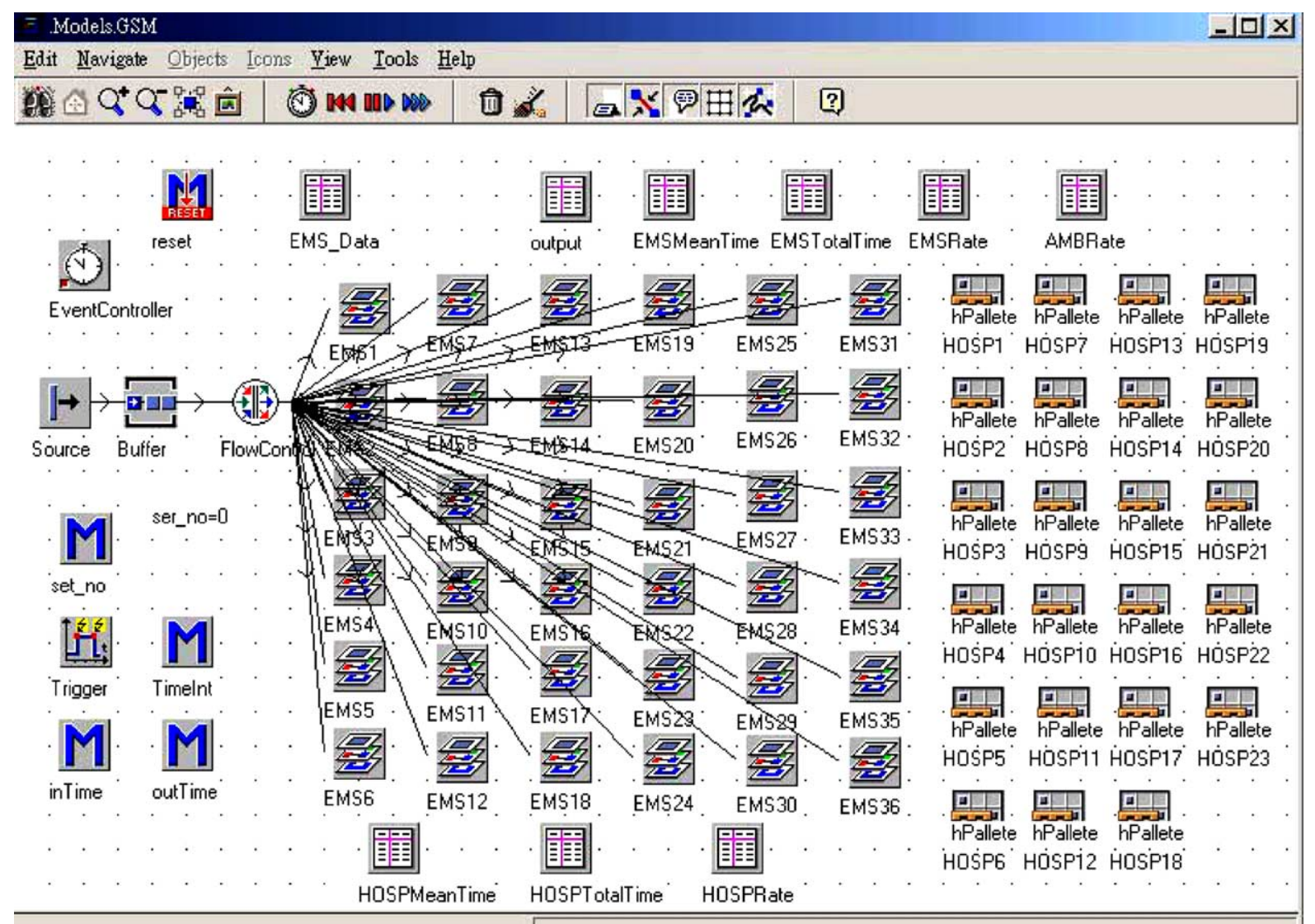

Fig. 2 The main system simulation model of the Taipei Emergency Medical Service System (two-tier rescue pattern) Screen display of software program eM-Plant, version 4.6.4. [28] The program is started with the Trigger object. Entities (event calls) are created in the Source object. The arrival times of three work shifts are entered. This activates the Timelnt object (method). The entities are then sent to the Buffer object to await entering the Flow Control object. The Flow Control object contains the probability distribution of dispatches across the 36 EMS subgroups (units). Based on this probability distribution, each entity is sent to its corresponding dispatcher, one of the EMS subgroups (EMS1 to EMS36). Event Controller object: coordinates and synchronizes the different events taking place during a simulation. reset object (method): reactivates a simulation model. Output, EMSMeanTime, EMSTotalTime, EMSRate, AMBRate, HOSPMeanTime, HOSPTotalTime, HOSPRate objects: the tables that carry the simulation output data. EMS_Data object: the basic input data for simulation modeling. HOSP1-HOSP23 objects: cooperative/assigned network hospitals set_no object (method): counts the number of entities (event calls) and their sequence. inTime, outTime object (method): gathers the start and end time of each rescue event segment.

- Alternative 1: One fixed network hospital was assigned to each emergency services subgroup to provide a two-tier rescue service. Each hospital's rescue unit was composed of one ALS-skilled medical team and one ambulance.

- Alternative 2: The number of two-tier rescue units (rescuers and ambulances) assigned to each network hospital was adjusted, based on the utilization rate and the probability of patients having to wait for rescue (due to the hospital not having a rescue team available). After the software simulated the various combinations of utilization rates and level of resources committed, the results were compared.
- Alternative 3: The cooperation team mix between the emergency services subgroup and the assigned network hospitals was changed, taking the proximity of the area into consideration. Each emergency services subgroup had the flexibility to cooperate with two to three network hospitals to provide two-tier rescue services. Each subgroup could arrange the dispatch sequence among the potentially cooperative hospitals in advance. When the first sequence was not available, then the second sequence performed the rescue. Based on this rule, three dispatch models were simulated separately and then compared: Dispatch Model 1-one fixed hospital, Dispatch 
Model 2- two hospitals in prearranged sequence, and Dispatch Model 3-three hospitals in prearranged sequence.

- Alternative 4 (Varied dispatch rate): Using the three dispatch models from above, the dispatch rate of the two-tier rescue was manipulated as 2 , 10 , and $20 \%$, respectively. The dispatch rate was defined as the percentage of runs to which a given ALS ambulance might be assigned. The probability of calls waiting and the utilization rates were then compared.

\section{Results}

\subsection{Empirical data}

The subject system performed a total of 6421 prehospital rescue jobs from 1-31 December 2000. Among those, 128 jobs (about 2\%) were a two-tier rescue. The current BLS personnel staffing levels, dispatch rates, and cooperative/assigned network hospitals across the 36 emergency services subgroups are revealed in Table 2 . The established

Table 2 Current personnel staffing level, rescue unit dispatch rate, and cooperative/assigned network hospitals across 36 emergency services subgroups

\begin{tabular}{|c|c|c|c|}
\hline $\begin{array}{l}\text { Emergency } \\
\text { services subgroup }\end{array}$ & $\begin{array}{l}\text { BLS personnel staffing level } \\
\text { (number of rescue teams) }\end{array}$ & $\begin{array}{l}\text { Dispatch } \\
\text { rate }(\%)\end{array}$ & $\begin{array}{l}\text { Cooperative/assigned } \\
\text { network hospitals }\end{array}$ \\
\hline EMS 1 & 2 & 4 & HOSP $10,13,6$ \\
\hline EMS 2 & 2 & 2.38 & HOSP $12,2,20$ \\
\hline EMS 3 & 1 & 1.14 & $\operatorname{HOSP} 12,19,23$ \\
\hline EMS 4 & 1 & 1.43 & $\operatorname{HOSP} 14,16,1$ \\
\hline EMS 5 & 1 & 0.67 & $\operatorname{HOSP} 19,23,20$ \\
\hline EMS 6 & 2 & 2.32 & $\operatorname{HOSP} 3,10,6$ \\
\hline EMS 7 & 2 & 5.87 & $\operatorname{HOSP} 1,23,14$ \\
\hline EMS 8 & 2 & 1.7 & HOSP 23, 19, 11 \\
\hline EMS 9 & 3 & 5.47 & $\operatorname{HOSP} 22,8,5$ \\
\hline EMS 10 & 1 & 2.03 & HOSP $5,8,3$ \\
\hline EMS 11 & 2 & 2.76 & $\operatorname{HOSP} 23,11,20$ \\
\hline EMS 12 & 2 & 2.29 & $\operatorname{HOSP} 23,19,20$ \\
\hline EMS 13 & 3 & 5.17 & HOSP $3,15,4$ \\
\hline EMS 14 & 2 & 1.46 & HOSP 2, 12, 23 \\
\hline EMS 15 & 1 & 1.73 & HOSP 5, 3, 15 \\
\hline EMS 16 & 2 & 1.4 & $\operatorname{HOSP} 16,1,9$ \\
\hline EMS 17 & 2 & 2.37 & HOSP 9, 3, 13 \\
\hline EMS 18 & 2 & 3.83 & HOSP $12,10,6$ \\
\hline EMS 19 & 1 & 4.3 & HOSP 20, 12, 19 \\
\hline EMS 20 & 3 & 3.24 & $\operatorname{HOSP} 3,5,15$ \\
\hline EMS 21 & 2 & 4.64 & HOSP 4, 9, 15 \\
\hline EMS 22 & 2 & 2.46 & HOSP $9,10,14$ \\
\hline EMS 23 & 1 & 2.13 & HOSP 5, 2, 8 \\
\hline EMS 24 & 2 & 1.39 & $\operatorname{HOSP} 12,2,5$ \\
\hline EMS 25 & 3 & 2.8 & HOSP $22,18,5$ \\
\hline EMS 26 & 2 & 1.31 & $\operatorname{HOSP} 5,12,2$ \\
\hline EMS 27 & 1 & 0.25 & $\operatorname{HOSP} 19,11$ \\
\hline EMS 28 & 2 & 3.33 & HOSP $12,10,5$ \\
\hline EMS 29 & 2 & 2.03 & $\operatorname{HOSP} 22,10,21$ \\
\hline EMS 30 & 1 & 0.45 & $\operatorname{HOSP} 20,19,23$ \\
\hline EMS 31 & 2 & 3.21 & $\operatorname{HOSP} 20,19,23$ \\
\hline EMS 32 & 2 & 2.69 & HOSP $8,5,1$ \\
\hline EMS 33 & 3 & 4.74 & $\operatorname{HOSP} 8,2,5$ \\
\hline EMS 34 & 2 & 7.58 & HOSP $8,5,7$ \\
\hline EMS 35 & 2 & 4.2 & HOSP $19,12,5$ \\
\hline EMS 36 & 1 & 1.23 & $\operatorname{HOSP} 17,19,23$ \\
\hline
\end{tabular}

Note: Currently each subgroup cooperates with one assigned (fixed) hospital and has the flexibility to cooperate with one or two additional cooperative network hospitals to provide two-tier rescue services. Each subgroup is equipped with one BLS rescue unit consisting of one rescue team (two EMS technicians) and one ambulance. BLS: basic life support. 


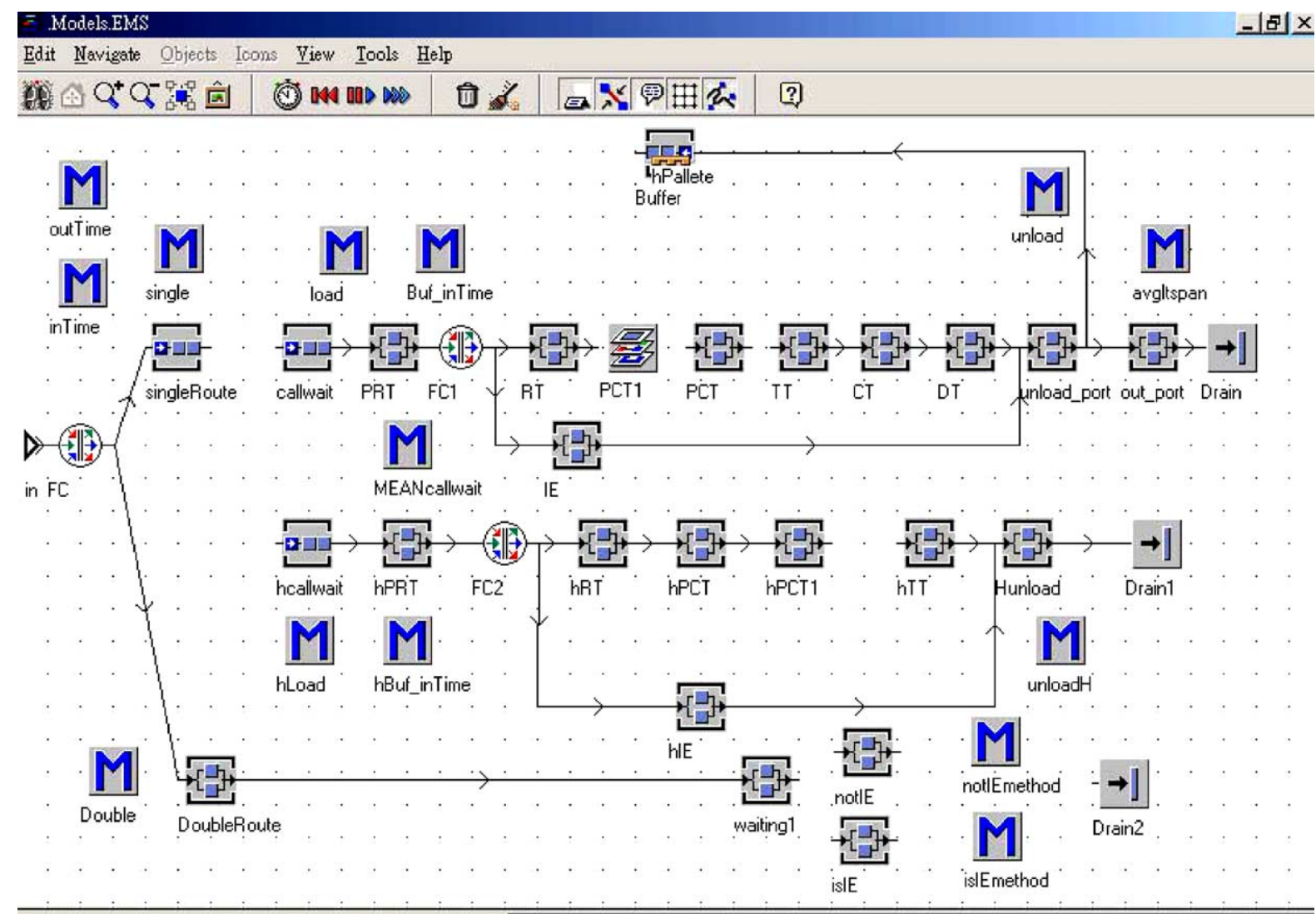

Fig. 3 Simulation model of 1 of 36 subsystems of the Taipei Emergency Medical Service System (two-tier rescue pattern) Screen display of software program eM-Plant, version 4.6.4. [28] This is an operational display example of one subgroup from the main system shown in Fig. 2. The subsystem describes the flowchart of each subgroup (unit). Entities (rescue event calls) enter the subsystem through the in object (interface), then go to the FC object, which contains the probability distributions of the one-tier- and two-tier system. For the 'one-tier system,' entities (rescue event calls) enter the singleRoute object, then activate the single object (method), then go to the callwait object that activates the callwait object method, a method to calculate the waiting time while the next process is still in busy. The Buffer1 object also activates the load object, a method to move the entity onto the pallete object (ambulance) and to load the pallete object (ambulance) from the Buffer object to the PRT object. Based on the probability distribution of actual and idle errands carried in the FC1 object, the entities (rescue event calls) go to process of RT-PCT1-PCT-TT-CT-DT or IE, respectively. They then enter the unload_port object, which activates the unload object, a method to unload entities to the out port object and to move the pallete objects (ambulances) back to the Buffer object. The out_port object also activates the avgltspan object, a method to calculate the average life span for each entity. The entities then terminate in the Drain object, which ends the subgroup's operation. For the 'two-tier system,' entities (rescue event calls) enter the DoubleRoute object, then activate The double object method, a method which create entities (rescue event calls) in the callwait object and the hcallwait object. The flowchart of entities (rescue event calls) which enter the callwait object is just same as the flowchart for the one-tier system; except the entities (rescue event calls) moving to PRT-RT-PCT1-PCT have to wait for those entering the hcallwait object to reach hPCT1 before moving to TT-CT-DT. The other entities (rescue event calls) enter the hcallwait object that activates the hBuf_inTime object, a method to calculate the waiting time while the next process is still in busy. The hcallwait object also activates the hload object, a method to move the entity onto the pallete object (ambulance) and to load the pallete object (ambulance) from one of the cooperative/assigned network hospitals (HOSP1-HOSP23) to the hPRT object. Based on the probability distribution of actual and idle errands carried in the $F C 2$ object, the entities (rescue event calls) go to the process of $h R T-h P C T$-hPCT then wait for the ones entering the callwait object to reach PCT, before move to $h T T$. They then enter the Hunload object, which activates the unloadH object, a method to unload entities to the Drain1 object and to move the pallete objects (ambulances) back to one of the cooperative/assigned network hospitals (HOSP1-HOSP23). The entities (rescue event calls) which enterered the hcallwait object then terminate in the Drain1 object. If the entities (rescue event calls) are idle errands (false alarm, not resulting in a rescue), they will activate the isIE object. inTime, outTime method: gathers the start time and end time of each entity (rescue event call). PRT object: preparation time-interval between the time the subgroup unit is notified and the time it responds by sending an ambulance rescue team. 
Table 3 Comparison of empirical data with simulation results for one- and two-tier rescues across 36 subgroups associated with 23 hospitals

\begin{tabular}{|c|c|c|c|c|c|c|c|c|}
\hline \multirow[t]{2}{*}{$\begin{array}{l}\text { Empirical and simulated } \\
\text { data }\end{array}$} & \multicolumn{2}{|c|}{$\begin{array}{l}\text { BLS event-site } \\
\text { arrival time (min) }\end{array}$} & \multicolumn{2}{|c|}{$\begin{array}{l}\text { ALS event-site } \\
\text { arrival time (min) }\end{array}$} & \multicolumn{2}{|c|}{$\begin{array}{l}\text { Patient hospital } \\
\text { arrival time (min) } \\
\end{array}$} & \multicolumn{2}{|c|}{$\begin{array}{l}\text { Turn-around } \\
\text { time }(\min ) \\
\end{array}$} \\
\hline & Mean & S.D. & Mean & S.D. & Mean & S.D. & Mean & S.D. \\
\hline \multicolumn{9}{|l|}{ Empirical data } \\
\hline One-tier only & 5.07 & 0.04 & & & 18.71 & 0.11 & 37.75 & 0.24 \\
\hline Two-tier hospital based & 5.07 & 0.04 & 5.35 & 0.06 & 19.95 & 0.27 & & \\
\hline \multicolumn{9}{|l|}{ Simulated data } \\
\hline One-tier rescue pattern & 6.00 & 0.09 & & & 20.89 & 0.04 & 37.72 & 0.59 \\
\hline Two-tier rescue pattern & 6.00 & 0.09 & 5.98 & 0.58 & 20.19 & 1.27 & & \\
\hline
\end{tabular}

Note: BLS, basic life support; ALS, advanced life support. Event-site arrival time: interval between the dispatching call and the BLS/ALS team's arrival on-site; hospital arrival time: period between dispatching call and patient's hospital arrival (includes the on-site rescue time); turn-around time: interval between the dispatching call and the BLS team's return back to its subgroup. S.D.: standard deviation.

system simulation model is shown in Figs. 2 and 3. The simulated results and the empirical data are presented in Table 3 and Student's $t$-test was performed with $0.05 \alpha$-level. There was no significant difference for ALS team event-site arrival time $(5.98 \pm 0.58 ; 5.35 \pm 0.06 \mathrm{~min})$, patient hospital arrival time $(20.19 \pm 1.27 ; 19.95 \pm 0.27 \mathrm{~min})$ and BLS team turn-around time $(37.72 \pm 0.59$; $37.75 \pm 0.24 \mathrm{~min}$ ) between the two data sets $(P<0.05)$.

\subsection{Simulated data}

\subsubsection{Alternatives 1 and 2}

We simulated Alternative 1 (one fixed network hospital responsible as each EMS' cooperative partner in a two-tier pattern) and took the current two-tier dispatch rate of $2 \%$ as simulation parame- ter (Table 4). Next, for Alternative 2, we identified the hospitals most likely to keep patients waiting (i.e., hospitals that get a dispatch order with no ambulance team available to go to the rescue) and labeled them as 22, 23, and 8 (Table 5). Among them, Hospital 22 was in a different location. In the hospital's $8 \mathrm{~min}$ driving distance, there was no other network hospital that could supplement the rescue task (Fig. 4). Therefore, for this specific hospital-in order to lessen waiting probability-we suggested two teams instead of one for its two-tier rescue. For Hospitals 23 and 8 , there were other network hospitals located in the same proximity to provide two-tier advanced life support services (Fig. 4). Thus, we did not recommend an elevation of resources for Hospitals 23 and 8 . After increasing Hospital 22's resources to two teams and simulating accordingly, the probability of calls waiting

hPRT object: preparation time of the cooperative/assigned network hospitals. $R T$ object: response timeinterval between the time the ambulance rescue team responds and the time it arrives at the scene. $h R T$ object: response time of the cooperative/assigned network hospitals. PCT1, PCT object: processing timeinterval between the time the ambulance rescue team arrives at the scene and treats the patient and the time it leaves the scene. hPCT1, hPCT object: processing time of the cooperative/assigned network hospitals. TT object: transport time-interval between the time the ambulance rescue team leaves the scene and arrives at hospital. hTT object: transport time of the cooperative/assigned network hospitals. CT object: caring time-interval between the time the ambulance rescue team arrives at the hospital and the time it leaves the hospital. DT object: departure time-interval between the time the ambulance rescue team leaves the hospital and the time it is back in service for the next rescue call. IE object: idle errand time-interval between the time the ambulance departs from the subgroup and returns to the subgroup without fulfilling a rescue. $h I E$ object: idle errand time of the cooperative/correspondent responsible hospitals. Idle errands may result from false alarms; fraudulent or prank calls; vague, insufficient descriptions given to the dispatcher preventing success in reaching the patient; too long a response time for patients to wait for the ambulance to arrive, and the possibility of having no ambulance available for rescue (which did not happen in empirical nor simulated data). 
Table 4 ALS dispatch rate, probability of calls waiting, and various time performances across alternative rescue patterns for 36 subgroups associated with 23 hospitals

\begin{tabular}{lcllll}
\hline $\begin{array}{l}\text { Empirical data and } \\
\text { simulated data with } \\
\text { various rescue patterns }\end{array}$ & $\begin{array}{l}\text { ALS } \\
\text { dispatch } \\
\text { rate (\%) }\end{array}$ & $\begin{array}{l}\text { Probability of } \\
\text { calls waiting } \\
(\%)\end{array}$ & $\begin{array}{l}\text { Mean call } \\
\text { waiting } \\
\text { time (min) }\end{array}$ & $\begin{array}{l}\text { Mean ALS } \\
\text { event-site arrival } \\
\text { time (min) }\end{array}$ & $\begin{array}{l}\text { Mean Patient } \\
\text { hospital arrival } \\
\text { time (min) }\end{array}$ \\
\hline $\begin{array}{l}\text { Empirical data (for 2-tier } \\
\quad \text { rescue only) }\end{array}$ & 2 & $-^{\mathrm{a}}$ & - & 5.35 & 19.95 \\
$\begin{array}{l}\text { Simulated data } \\
\quad \text { One-tier rescue }\end{array}$ & & & & \\
$\quad \begin{array}{l}\text { Two-tier rescue } \\
\text { With one fixed, }\end{array}$ & 2 & 2.4 & 0.54 & 5.98 & 21.26 \\
$\quad$ responsible network & 10 & 4.4 & 0.80 & 6.09 & 20.19 \\
$\quad$ hospital per subgroup & 20 & 6.9 & 1.52 & 6.49 & 20.20 \\
$\quad$ As above, but with & 2 & 1.1 & 0.36 & 5.70 & 21.47 \\
$\quad$ elevated resource & 10 & 2.5 & 0.63 & 6.06 & 20.59 \\
$\quad$ level for Hospital 22 & 20 & 4.9 & 1.06 & 6.48 & 21.26 \\
\hline
\end{tabular}

Note: In the simulation, the ALS dispatch rate was manipulated as 2, 10, and $20 \%$. ALS: advanced life support. Call waiting: time interval between patient's emergency call, the dispatcher assigning a specific EMS subgroup (with corresponding network hospital) to the rescue, plus any potential waiting time if the ambulance rescue team is currently occupied with another job (only simulated call waiting data are available for BLS and ALS). Event-site arrival time: interval between the dispatching call and the BLS/ALS team's arrival on-site; hospital arrival time: period between dispatching call and the patient's hospital arrival (includes the on-site rescue time).

${ }^{\text {a }}$ Data not available.

${ }^{\mathrm{b}}$ For Hospital 22, the number of ALS rescue teams was increased from one to two teams.

Table 5 Incidence of call waiting for ALS rescue pattern with one fixed response network hospital among 23 hospitals

\begin{tabular}{lrlll}
\hline & Dispatch No. & Call waiting No. & Call waiting incidence & Percentage of call waiting \\
\hline Hospital 1 & 21 & 0 & 0.00 & 0.00 \\
Hospital 2 & 21 & 0 & 0.00 & 0.00 \\
Hospital 3 & 145 & 1 & 0.01 & 0.03 \\
Hospital 4 & 30 & 0 & 0.00 & 0.00 \\
Hospital 5 & 71 & 1 & 0.01 & 0.03 \\
Hospital 6 & 0 & 0 & 0.00 & 0.00 \\
Hospital 7 & 0 & 0 & 0.00 & 0.00 \\
Hospital 8 & 181 & 3 & 0.02 & 0.10 \\
Hospital 9 & 72 & 0 & 0.00 & 0.00 \\
Hospital 10 & 48 & 0 & 0.00 & 0.00 \\
Hospital 11 & 0 & 0 & 0.00 & 0.00 \\
Hospital 12 & 130 & 1 & 0.00 & 0.03 \\
Hospital 13 & 0 & 0 & 0.00 & 0.00 \\
Hospital 14 & 9 & 0 & 0.00 & 0.00 \\
Hospital 15 & 0 & 0 & 0.00 & 0.00 \\
Hospital 16 & 29 & 0 & 0.00 & 0.00 \\
Hospital 17 & 18 & 0 & 0.00 & 0.00 \\
Hospital 18 & 0 & 0 & 0.00 & 0.00 \\
Hospital 19 & 133 & 0 & 0.00 & 0.03 \\
Hospital 20 & 84 & 1 & 0.01 & 0.00 \\
Hospital 21 & 0 & 0 & 0.00 & 0.69 \\
Hospital 22 & 66 & 20 & 0.30 & 0.07 \\
Hospital 23 & 157 & 2 & 0.01 & 1.00 \\
Total & 1215 & 29 & 0.02 & \\
\hline
\end{tabular}


A. HOSPITAL 22

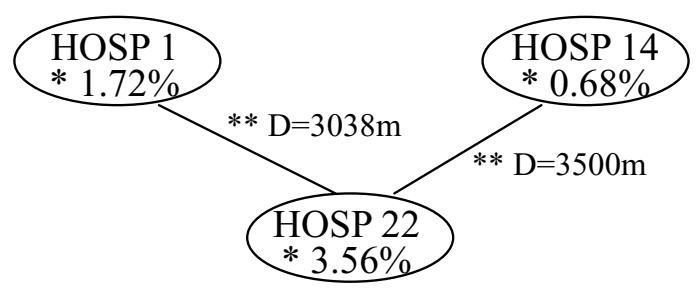

B. HOSPITAL 8

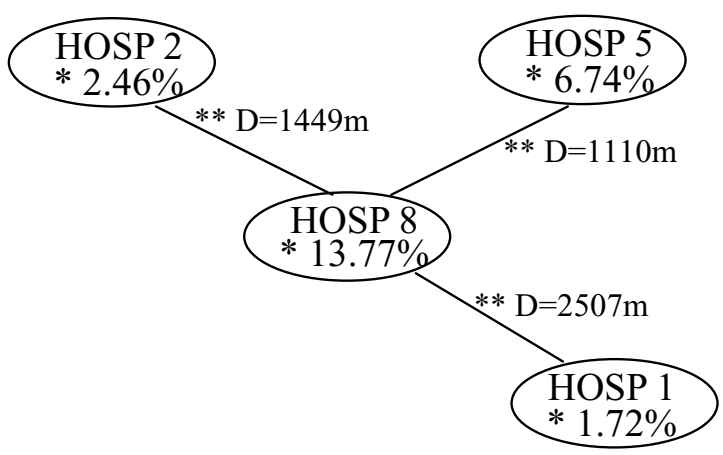

C. HOSPITAL 23

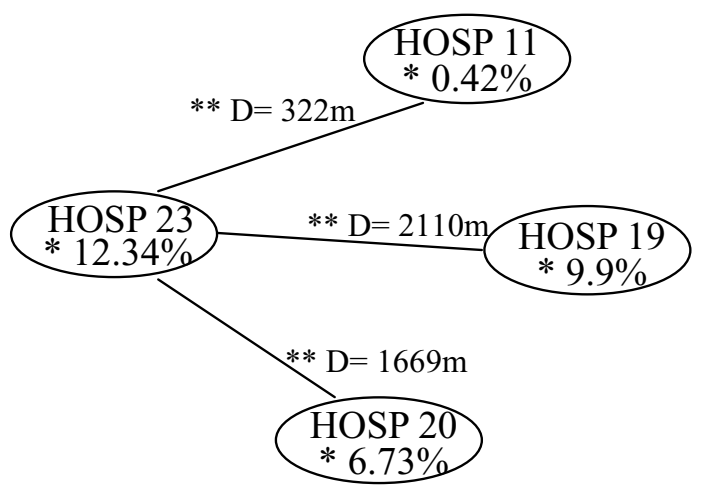

The distance between HOSPITAL 22 with its backup hospitals were $3500 \mathrm{~m}$ and $3038 \mathrm{~m}$ greater than that of HOSPITAL 8 with its backup hospitals $(2507 \mathrm{~m}, 1449 \mathrm{~m}$ and $1110 \mathrm{~m})$ and greater that that of HOSPITAL 23 with its backup hospitals $(2110 \mathrm{~m}$, $1669 \mathrm{~m}$ and $322 \mathrm{~m}$ )

* dispatch rate

** distance between responsible hospital and its backup hospitals

Fig. 4 Geographical map of Hospital 22, 8, 23 (the first three leading hospitals with highest waiting probability) with possible cross-coverage hospitals. 
Table 6 Simulated performances for the two-tier rescue pattern across different dispatch models and dispatch rates

\begin{tabular}{|c|c|c|c|c|c|c|c|c|c|c|}
\hline \multirow[t]{2}{*}{$\begin{array}{l}\text { Number of cooperative } \\
\text { network hospitals per } \\
\text { EMS subgroup }^{\mathrm{a}}\end{array}$} & \multirow[t]{2}{*}{$\begin{array}{l}\text { Dispatch rate of } \\
\text { two-tier rescue } \\
\text { pattern (\%) }\end{array}$} & \multirow[t]{2}{*}{$\begin{array}{l}\text { Probability of call } \\
\text { waiting more than } \\
8 \text { min (\%) }\end{array}$} & \multirow[t]{2}{*}{$\begin{array}{l}\text { Probability of } \\
\text { call waiting } \\
\text { (\%) }\end{array}$} & \multicolumn{2}{|c|}{$\begin{array}{l}\text { Call Waiting } \\
\text { time (min) }\end{array}$} & \multicolumn{2}{|c|}{$\begin{array}{l}\text { ALS event-site } \\
\text { arrival time } \\
\text { (min) } \\
\end{array}$} & \multicolumn{2}{|c|}{$\begin{array}{l}\text { Patient hospital } \\
\text { arrival time } \\
\text { (min) }\end{array}$} & \multirow[t]{2}{*}{$\begin{array}{l}\text { Utilization rate } \\
\text { of ALS rescue } \\
\text { (\%) }\end{array}$} \\
\hline & & & & Mean & S.D. & Mean & S.D. & Mean & S.D. & \\
\hline $1^{b}$ & $\begin{array}{r}2 \\
10 \\
20\end{array}$ & $\begin{array}{l}2.22 \\
3.11 \\
5.15\end{array}$ & $\begin{array}{l}2.38 \\
4.36 \\
6.86\end{array}$ & $\begin{array}{l}0.54 \\
0.80 \\
1.52\end{array}$ & $\begin{array}{l}0.63 \\
0.30 \\
0.68\end{array}$ & $\begin{array}{l}5.98 \\
6.09 \\
6.49\end{array}$ & $\begin{array}{l}0.58 \\
0.33 \\
0.28\end{array}$ & $\begin{array}{l}20.19 \\
20.20 \\
20.87\end{array}$ & $\begin{array}{l}1.27 \\
0.61 \\
0.59\end{array}$ & $\begin{array}{l}0.35 \\
1.70 \\
3.42\end{array}$ \\
\hline $2^{c}$ & $\begin{array}{r}2 \\
10 \\
20\end{array}$ & $\begin{array}{l}0.08 \\
0.10 \\
0.24\end{array}$ & $\begin{array}{l}0.08 \\
0.15 \\
0.52\end{array}$ & $\begin{array}{l}0.04 \\
0.02 \\
0.05\end{array}$ & $\begin{array}{l}0.24 \\
0.05 \\
0.05\end{array}$ & $\begin{array}{l}5.53 \\
5.40 \\
5.43\end{array}$ & $\begin{array}{l}0.45 \\
0.20 \\
0.14\end{array}$ & $\begin{array}{l}19.78 \\
19.69 \\
19.73\end{array}$ & $\begin{array}{l}1.33 \\
0.61 \\
0.40\end{array}$ & $\begin{array}{l}0.35 \\
1.74 \\
3.47\end{array}$ \\
\hline $3^{d}$ & $\begin{array}{r}2 \\
10 \\
20\end{array}$ & $\begin{array}{l}0.00 \\
0.00 \\
0.01\end{array}$ & $\begin{array}{l}0.00 \\
0.00 \\
0.02\end{array}$ & $\begin{array}{l}0.00 \\
0.00 \\
0.00\end{array}$ & $\begin{array}{l}0.00 \\
0.00 \\
0.01\end{array}$ & $\begin{array}{l}5.42 \\
5.43 \\
5.35\end{array}$ & $\begin{array}{l}0.49 \\
0.18 \\
0.13\end{array}$ & $\begin{array}{l}19.16 \\
19.69 \\
19.56\end{array}$ & $\begin{array}{l}1.18 \\
0.41 \\
0.42\end{array}$ & $\begin{array}{l}0.34 \\
1.71 \\
3.42\end{array}$ \\
\hline
\end{tabular}

Note: S.D., standard deviation; ALS, advanced life support. Call waiting: time interval between patient's emergency call, the dispatcher assigning a specific EMS subgroup (with corresponding network hospital) to the rescue, plus any potential waiting time if the ambulance rescue team is currently occupied with another job (only available for simulated BLS/ALS data); event-site arrival time: interval between the dispatching call and the BLS/ALS team's arrival on-site; hospital arrival time: period between dispatching call and the patient's hospital arrival (includes the on-site rescue time).

${ }^{a}$ In this simulation, the dispatch rate was manipulated to be 2, 10, and 20\% for Dispatch Models 1-3.

b Dispatch Model 1: one fixed network hospital per subgroup.

' Dispatch Model 2: two fixed hospitals per subgroup in prearranged dispatch sequence.

${ }^{d}$ Dispatch Model 3: three fixed hospitals per subgroup in prearranged dispatch sequence. 
lessened by about $50 \%$ (i.e., from 2.4 to $1.1 \%$ ) (see Table 4). Even when the simulated dispatch rates of the two-tier rescue were increased to 10 and $20 \%$, the probabilities of calls waiting were 2.5 and $4.9 \%$, respectively. The event-site arrival times, on-site treatment times, and hospital arrival times remained the same (see Table 4).

\subsubsection{Alternatives 3 and 4}

The operating rule of Dispatch Model 2 is that when an EMS gets a rescue call-and a two-tier model is assessed as necessary-the ALS team of the responsible hospital of the first sequence is dispatched for the job; if it is already on duty, then the responsible hospital of the second sequence is dispatched. If that is also still on a job, patients have to wait. Alternatively, Dispatch Model 3 in- creases the number of cooperative/responsible hospitals to three and follows the same rules as Dispatch Model 2. We simulated the latter two dispatch models and compared the results with those derived from Dispatch Model 1 (one fixed hospital). The outcomes are revealed in Table 6. They show that by changing the cooperation pattern from one fixed, responsible hospital to two cooperative hospitals per subgroup (Dispatch Model 2), the call waiting probability can be lowered from $2.38 \%$ (at a dispatch rate of $2 \%$ ) to less than $1 \%$ for any of the three simulated dispatch rates of 2 , 10 , and $20 \%$. When the cooperation pattern was changed to Dispatch Model 3 (three cooperative hospitals per subgroup), the call waiting probability decreased to less than $0.05 \%$. Moreover, the response time and hospital arrival time of Dispatch

Table 7 Intervention time performances of various life support techniques across different rescue patterns

\begin{tabular}{|c|c|c|c|c|c|}
\hline \multirow[t]{2}{*}{ BLS intervention time } & \multicolumn{5}{|c|}{$\begin{array}{l}\text { Occurrence of specific ALS intervention times (\%) } \\
\text { (event-site arrival times) }\end{array}$} \\
\hline & Model $A^{a}$ & Model $\mathrm{B}^{\mathrm{b}}$ & Model $\mathrm{C}^{\mathrm{C}}$ & Model $\mathrm{D}^{\mathrm{d}}$ & Model $\mathrm{E}^{\mathrm{e}}$ \\
\hline \multicolumn{6}{|l|}{ ALS intervention less than $8 \mathrm{~min}$} \\
\hline$<4 \min$ & 2.2 & 25.24 & 22.96 & 22.97 & 23.01 \\
\hline $4-8 \min$ & 0.76 & 41.23 & 47.6 & 46.78 & 46.86 \\
\hline$>8 \min$ & 0 & 17.07 & 14.66 & 16.87 & 17.08 \\
\hline Total & 2.96 & 83.54 & 85.22 & 86.62 & 86.95 \\
\hline \multicolumn{6}{|l|}{ ALS intervention $8-16 \mathrm{~min}$} \\
\hline$<4 \min$ & 12.09 & 3.25 & 4.33 & 3.16 & 3.4 \\
\hline $4-8 \min$ & 18.87 & 8.41 & 7.33 & 7.11 & 6.61 \\
\hline$>8 \min$ & 2.01 & 2.4 & 2.4 & 2.55 & 2.35 \\
\hline Total & 32.97 & 14.06 & 14.06 & 12.82 & 12.36 \\
\hline \multicolumn{6}{|l|}{ ALS intervention greater than 16 min } \\
\hline$<4 \min$ & 12.6 & 0.48 & 0.24 & 0.21 & 0.27 \\
\hline $4-8 \min$ & 34.13 & 1.44 & 0.24 & 0.26 & 0.3 \\
\hline$>8 \mathrm{~min}$ & 17.34 & 0.48 & 0.24 & 0.09 & 0.12 \\
\hline Total & 64.07 & 2.4 & 0.72 & 0.56 & 0.69 \\
\hline $\begin{array}{l}\text { Total \% occurrence of all intervention } \\
\text { times per model }\end{array}$ & 100 & 100 & 100 & 100 & 100 \\
\hline
\end{tabular}

Note: All data include an increase in ALS resources for Hospital 22 from one rescue team to two. BLS: basic life support; ALS: advanced life support. Intervention time (event-site arrival time): interval between the dispatching call and the BLS/ALS team's arrival on-site.

${ }^{a}$ Model A: one-tier rescue model-the hospital arrival time is taken as the ALS intervention time.

${ }^{b}$ Model B: two-tier rescue model-assumes that each emergency services subgroup (EMS) cooperates with one fixed network hospital (Dispatch Model 1 ) with a $2 \%$ two-tier dispatch rate.

c Model C: two-tier rescue model-assumes that each EMS cooperates with two fixed network hospitals (Dispatch Model 2) with a $2 \%$ dispatch rate.

d Model D: two-tier rescue model-assumes that each EMS cooperates with two fixed network hospitals (Dispatch Model 2) with a $10 \%$ dispatch rate.

e Model E: two-tier rescue model-assumes that each EMS cooperates with two fixed network hospitals (Dispatch Model 2) with a $20 \%$ dispatch rate. 
S. Su, C.-L. Shih

Model $2(5.53 \pm 0.45 ; 19.78 \pm 1.33 \mathrm{~min})$ and Dispatch Model $3(5.42 \pm 0.49 ; 19.16 \pm 1.18 \mathrm{~min})$ were less than that of Dispatch Model 1 (5.98 \pm 0.58 ; $20.19 \pm 1.27 \mathrm{~min})$. The outcomes remained the same when the two-tier dispatch rates were changed to 10 and 20\% (Table 6).

Based on the literature [20], if patients with cardiac arrest receive BLS treatment within $4 \mathrm{~min}$ and initiate ALS treatment (CPR) within $8 \mathrm{~min}$, they have a higher successive resuscitation rate. Thus, we set a $4 \mathrm{~min}$ arrival time as the criterion for the one-tier rescue pattern, and $8 \mathrm{~min}$ as the criterion for the two-tier rescue pattern. We staffed Hospital 22 with two rescue teams instead of one. We then compared the achievement rate of the different rescue patterns. The results are shown in Table 7. For the two-tier system, the achievement rate was $25 \%$ for Model B (Dispatch Model 1-one fixed hospital per subgroup-with a $2 \%$ dispatch rate. When we took Model C (Dispatch Model 2-two fixed hospitals per subgroup-with a $2 \%$ dispatch rate), the achievement rate was $23 \%$. If the dispatch rate of Dispatch Model 2 increased to $10 \%$ and $20 \%$ (Models D and E), the achievement rate still remained around $23 \%$. Therefore, we suggested to increase Hospital 22's staffing level and to use the Dispatch Model 2 cooperation model (two fixed hospitals per subgroup) as the most cost-effective alternative to perform the two-tier rescue policy.

\subsection{Summary}

If one put the two-tier rescue model aside and only considered the one-tier rescue model (Model A), the 4 min BLS site arrival/8 min ALS site arrival probability was about $2.2 \%$. However, the optimum solution was obtained if the subject system performed a two-tier rescue pattern using Dispatch Model 2 (two fixed, responsible hospitals per subgroup), took proximity into account in selecting specific hospitals for the subgroups, and enhanced the resource level (personnel teams and ambulance) of Hospital 22 from one to two. This resulted in a less than $1 \%$ calls waiting probability, no delays in patients' hospital arrival, an achievement rate of $23 \%$ for the 4 min BLS site arrival/ 8 min ALS site arrival probability, and the lowest resource commitment situation.

This research also found a low utilization rate of the two-tier rescue. Therefore, it was recommended that dispatchers receive more training to increase utilization. The current staffing level can still afford the elevated dispatch rate of a two-tier rescue system without interfering with its service quality.

\section{Discussion}

\subsection{Simulation models: definition and use}

Simulation tool is used to describe and analyze the behavior of a system, ask what-if questions about the real system, and aid in the design of a real system [21]. The simulation practice often "utilize(s) symbols to represent different parts of the system, enhance the mutual understanding of the interaction and relationship, simultaneously those symbols can be integrated to set up a model to mimic the real system, and hence predict the system behavior [22]."' Among different models, the mimic model is considered to be a performance evaluation tool. It generally uses a computer to simulate system behavior, to reveal a causal relationship existing in the real world, and to evaluate performance, such as the resource utilization rate, turn-around time, waiting time, etc. It also can be used to predict the outcome after changing some parameters and to provide a reference for decision making. To construct a simulation model is more difficult. Hopefully, various recently developed simulation software packages will make simulation model construction easier than before. With a strong operating capacity, the simulation can proximate the real world. So far, many areas have used simulation methodology to solve complicated problems, such as military simulation for fighting strategies, arms distribution simulation, logistics, transportation and communication simulation, services system simulation for process reengineering, on-line simulation, and resource management simulation. Distribution system simulation covers the range of manufacturing process simulation, performance evaluation, circle time simulation, among others. It was not until the mid-1990s, that the health care system simulation gained important attention for using simulation methodology to execute total quality management or continuous quality improvement, resource management, etc. [23-27]. Other fields, such as transportation systems and production systems, have also used simulation successfully. Development of this information technology made on-line simulation possible. In combination with other interfaces, it makes real-world simulation more effective.

\subsection{Implications and applications of the developed simulation model}

Prehospital care is one of the main responsibilities of the emergency services system. Especially for prehospital cardiac arrest patients with ventricular 
fibrillation and ventricular tachycardia, appropriate and speedy rescue greatly affects patients' prognosis. If automatic external defibrillation can be performed earlier, the survival rate is increased $[6,7,28]$. As to which kind of rescue pattern can attain the best prognosis, much research has found time to be the most important factor affecting the prognosis of out-of-hospital cardiac arrest patients. Therefore, earlier intervention of basic life support and defibrillation are two significant factors [13].

For this subject system, simulated data showed the following: for the one-tier rescue model, the average arrival time at the scene (i.e., the starting time of basic life support) was about $6 \mathrm{~min}$ ( $5 \mathrm{~min}$ empirical). Hospital arrival time (period between the dispatching call and patient's arrival at the hospital) was simulated as $21 \mathrm{~min}(19 \mathrm{~min}$ in empirical) (see Table 3). That means, if the advanced life support technique can be executed only after patients arrived at the hospital, those prehospital cardiac arrest patients have to wait about $21 \mathrm{~min}$ to receive ALS care and thus lose the best timing for treatment. The two-tier rescue pattern for the subject system is aimed at providing hospital-based, in-time, advanced life support services (i.e., administered within the most critical first $8 \mathrm{~min}$ ). Since the ALS event-site arrival time for the two-tier rescue pattern averages close to $6 \mathrm{~min}$, this means that ALS can intervene about 15 min earlier than the one-tier rescue pattern to improve the prognosis of prehospital cardiac arrest patients. These systems require more research to fully understand their exact impacts. The subject system took hospitals' medical care personnel as the two-tier rescuers. Undoubtedly, the cost of this system is quite high. Therefore, the main concern of policy makers is how to perform the two-tier rescue pattern at the lowest possible cost with a brief, simple dispatching pattern while still achieving maximum services and minimal waiting time.

\subsection{Conclusions, limitations, and summary}

This research applied the simulation method and tested several potential operating models. It took the current 23 network hospitals and 36 EMS subgroups as its sample. The simulation model established the following as optimum: (1) although the differences were not great, Dispatch Model 2 (two hospitals per subgroup) of the two-tier rescue model is recommended. It can decrease the waiting probability and waiting time as well as enhance proper medical treatment. In addition, it streamlines the dispatching process and decreases randomity. Each EMS should cooperative with two selected network hospitals in a prearranged dispatch sequence (Dispatch Model 2) to simplify the dispatcher's decision procedures while facing the situation that the two-tier rescue pattern requires. (2) Hospital 22's resource level should be increased from one rescue team to two. The model suggested this special treatment for Hospital 22 because simulation outcomes showed that it was uniquely located. If another hospital were assigned as its backup, a deferred response could occur. With the new, suggested operational pattern, the probability of waiting was the lowest. The two-tier rescue team could reach the event-site in $6 \mathrm{~min}$ to provide treatment. On the other hand, the utilization rate of the two-tier rescue pattern was still low (about $2 \%$ with simulation forecasting. If the two-tier rescue utilization rates were 10 or $20 \%$, based on the above-recommended operational pattern, the service performance could still be maintained.

In accordance with the criteria (that basic life support intervene within $4 \mathrm{~min}$, and advanced life support be executed in $8 \mathrm{~min}$ ), no matter which pattern of two-tier rescue was used, the achievement rate of two-tier rescue pattern was 10 times the one-tier rescue model (Table 7). If costs were not the subject, the two-tier rescue pattern would obviously surpass the one-tier rescue pattern. However, if we explore more deeply the content of the two-tier rescue pattern and try to understand how intervention with two-tier rescue actually affects the prognosis elevation for the casualties, we may also consider whether any operation pattern could achieve a similar, positive effect. An example would be automatic external defibrillation for ventricular fibrillation or ventricular tachycardia patients. Another factor to improve the prognosis of patients might be to increase the utilization rate of the two-tier rescue pattern.

The limitation of the current study is its restricted database. Therefore, no seasonal variation could be included. This can be remedied in future, expanded studies.

In summary, the simulation method applied in this research showed its merits of being low cost, low risk, and it is easily manipulable and useful for other emergency services systems in rescue model developing.

\section{Acknowledgements}

The authors received financial support from the National Science Council (NSC 89-2314-B-002-383), Taiwan. 


\section{References}

[1] N. Urban, L. Bergner, M.S. Eisenberg, The costs of a suburban paramedic program in reducing deaths due to cardiac arrest, Med. Care 4 (1981) 379-392.

[2] T.D. Valenzuela, E.A. Criss, D. Spaite, et al., Costeffectiveness analysis of paramedic emergency medical services in the treatment of prehospital cardiopulmonary arrest, Ann. Emerg. Med. 19 (1990) 1407-1411.

[3] G. Riediger, T.F. Sperber, Efficiency and cost-effectiveness of advanced EMS in West Germany, Am. J. Emerg. Med. 8 (1990) 76-80.

[4] A. Hallstrom, M.S. Eisenberg, L. Bergner, Modeling the effectiveness and cost-effectiveness of an emergency service system, Soc. Sci. Med. 15C (1981) 13-17.

[5] G. Nichol, A.S. Detsky, I.G. Stiell, K. O’Rourke, G. Wells, A. Laupacis, Effectiveness of emergency medical services for victims of out-of-hospital cardiac arrest: a meta analysis, Ann. Emerg. Med. 27 (1996) 700-710.

[6] R.D. White, B.R. Asplin, T.F. Bugliosi, D.G. Hankins, High discharge survival rate after out-of-hospital ventricular fibrillation with rapid defibrillation by police and paramedics, Ann. Emerg. Med. 28 (1996) 480-485.

[7] C.S. Forrer, R.A. Swor, R.E. Jackson, R.G. Pascual, S. Compton, C. McEachin, Estimated cost-effectiveness of a police automated external defibrillator program in a suburban community: 7 years experience, Resuscitation 52 (2000) 23-29.

[8] G. Nichol, A.P. Hallstrom, J.P. Ornato, B. Riegel, I.G. Stiell, T. Valenzuela, G.A. Wells, R.D. White, M.L. Weisfeldt, Potential cost-effectiveness of public access defibrillation in the United States, Circulation 97 (1998) 1315-1320.

[9] M.S. Eisenberg, B.T. Horwood, R.O. Cummins, R. ReynoldsHaertle, T.R. Hearne, Cardiac arrest and resuscitation: a tale of 29 cities, Ann. Emerg. Med. 19 (1990) 179-186.

[10] M.P. Larsen, M.S. Eisenberg, R.O. Cummins, A.P. Hallstrom, Predicting survival from out-of-hospital cardiac arrest: a graphic model, Ann. Emerg. Med. 22 (1993) 1652-1658.

[11] T.D. Valenzuela, D.J. Roe, S. Cretin, D.W. Spaite, M.P. Larsen, Estimating effectiveness of cardiac arrest interventions: a logistic regression survival model, Circulation 96 (1997) 3308-3313.

[12] R.O. Cummins, From concept to standard-of-care? Review of clinical experience with automated external defibrillators, Ann. Emerg. Med. 18 (1989) 1269-1275.

[13] R.O. Cummins, M.S. Eisenberg, A.P. Hallstrom, P.E. Litwin, Survival of out-of-hospital cardiac arrest with early initiation of cardiopulmonary resuscitation, Am. J. Emerg. Med. 3 (1985) 114-119.
[14] N. Graham, A. Laupacis, I.G. Stiell, K. O’Rourke, A. Anis, H. Bolley, A.S. Detsky, Cost-effectiveness analysis of potential improvements to emergency medical services for victims of out-of-hospital cardiac arrest, Ann. Emerg. Med. 27 (1996) 711-720.

[15] L. Moore, Measuring quality and effectiveness of prehospital EMS, Prehosp. Emerg. Care 3 (4) (1999) 325331.

[16] Guidelines 2000 for cardiopulmonary resuscitation and emergency cardiovascular care. An international consensus on science, Suppl. Circ. 102 (2000) 1-363.

[17] eM-Plant Software, Version 4.6.4, Tecnomatix Technologies, Ltd., Neu-Isenburg, Germany.

[18] D. Spaite, R. Benoit, D. Brown, R. Cales, D. Dawson, C. Glass, C. Kaufmann, D. Pollock, S. Ryan, E.M. Yano, Uniform prehospital data elements and definitions: a report from the uniform prehospital emergency medical services data conference, Ann. Emerg. Med. 25 (1995) 525534.

[19] BestFit Software, Version 2.0, Palisades Corporation, Newfield, NY, USA.

[20] M.S. Eisenberg, L. Bergner, A. Hallstrom, Cardiac resuscitation in the community. Importance of rapid provision and implication for program planning, JAMA 241 (1979) 1905-1907.

[21] J. Banks, Handbook of Simulation: Principles, Methodology, Advances, Applications and Practice, first ed., Wiley, New York, NY, 1998, pp. 3-30.

[22] C. Harrell, K. Tumay, Simulation Made Easy: A Manager's Guide, fifth ed., Elle Snodgrass, Norcross, GA, 1999, pp. $26-31$.

[23] N. Ohboshi, H. Masui, Y. Kambayashi, A study of medical emergency workflow, Comput. Methods Prog. Biomed. 55 (3) (1998) 177-190.

[24] C.E. Saunders, P.K. Makens, L.J. Leblanc, Modeling emergency department operations using advanced computer simulation systems, Ann. Emerg. Med. 18 (2) (1989) 134140.

[25] F. McGuire, Using simulation to reduce length of stay in emergency department, JSHS 5 (3) (1997) 81-90.

[26] F. Hashimoto, S. Bell, Improving outpatient clinic staffing and scheduling with computer simulation, J. Gen. Intern. Med. 11 (1996) 182.

[27] L. Chin, G. Fleisher, Planning model of resource utilization in an academic pediatric emergency department, Ped. Emerg. Care 14 (1) (1998) 4-9.

[28] R.E. Kerber, Statement on early defibrillation from the Emergency Cardiac Care Committee, American Heart Association, Circulation 83 (1991) 2233.

Available online at www.sciencedirect.com

science@direct. 